\title{
Using group decision support systems in the preparation of real estate development projects
}

\author{
Elena Gusakova* and Elena Romanova ${ }^{\dagger}$ \\ Moscow State University of Civil Engineering, Yaroslavskoe shosse, 26, Moscow, 129337, Russia
}

\begin{abstract}
The preparation and implementation of a real estate development project requires the adoption of a large number of diverse management decisions. For the preparation of decisions often attract experts. The opinions of many experts sometimes do not agree with each other and even are mutually exclusive. For their analysis using computerbased decision support systems (DSS). The article provides an analysis of several DSS, their applicability for the preparation of the project of real estate development. The results of the comparative experiment are also presented - the advantages and disadvantages of direct and indirect discussion of the project. It is revealed that the mediated written discussion with the use of information and communication technologies has advantages over direct discussion. It has been established that the quality of the discussion improves when there is a specially trained moderator who controls the process. Analysis of the features of the implementation of real estate development projects showed that there are no group decision support systems on the software market that can meet all the requirements necessary for the effective preparation and implementation of a real estate development project.
\end{abstract}

\section{Introduction}

In managerial practice, a group solution is gaining more and more weight as the complexity and scope of the problems being solved increase. The scope and the large number of participants are a characteristic feature of real estate development projects. They include not only a fundamental physical change of the property, but also significant economic, legal and social effects in the development area [1]. During the implementation of such projects, an increasing number of specialists and experts are grouped together to integrate opinions and make common decisions on the project.The integration of judgments and the experience of experts is not determined by their own desire, but by objective necessity. Competent opinion is required in all issues and areas covered by the project - to solve many design, organizational, industrial, technological, legal, urban planning, social and other tasks. Obviously, this is beyond the power of one person $[2,3,4]$.

From the point of view of management and the development of collective decisions, grouping has both positive and negative effects $[5,6]$.

\footnotetext{
* Corresponding authors: GusakovaEA@mgsu.ru

† RomanovaEV@mgsu.ru
} 
Positive sides:

- A large stock of diverse knowledge. The group as a whole always has more knowledge than the most authoritative and informed specialist of this group;

- The greatest number of opinions and points of view. Solving development problems requires a variety of approaches and types of thinking. But one person usually sees only one side of the problem. The more diverse and diverse the primary experience of the participants, the higher the likelihood of the formation of an innovative solution;

- High activity of the participants. In a group, people, as a rule, have a desire to share their own opinions and refute the distorted information. A group decision includes comparing the different opinions of its participants, defending their points of view, competing for a greater contribution to solving the problem.

Negative sides:

- The pressure of the majority. If a large number of participants in the group hold the same opinion, it is not possible to convince them and promote another solution. In order to be accepted by the group, some participants have to give up their opinions;

- The primacy of a strong personality. A person with a higher status or having good oratorical abilities is able to oppress other participants. He suppresses other people's statements, takes most of the time itself and thereby suppresses the initiative;

- Reduced sense of reality. Group solutions are always more risky than individual ones. Since the group solution was developed jointly, it seems to be the best. In addition, each participant disclaims personal responsibility for the process and outcome of the work [7].

Modern computerized decision support systems help to use the most positive effects of group work and at the same time minimize its negative psychological effects $[8,9]$.

Decision Support System (DSS) - a complex of mathematical and heuristic methods and models to substantiate the choice of the most appropriate management decision.

DSS has the following four main characteristics:

1) uses both data and models;

2) helps to make decisions in semistructured and unstructured tasks;

3) does not give ready-made decisions, but helps to justify them and supports their adoption;

4) increases the efficiency of decisions $[10,11]$.

To manage a real estate development project, it is important that there are two types of DSS: operational and strategic. Operational DSS are designed to immediately respond to changes in the current situation in the management of financial and business processes of the project. Strategic DSSs focused on the analysis of large volumes of heterogeneous information collected from various sources. These DSSs help to find and justify the most rational options for real estate development, taking into account the influence of significant direct and indirect factors, such as the likely direction of development of the building area, public opinion, the situation of target and financial markets, changes in legislation and many others $[12,13]$.

DSS of the first type are called Executive Information Systems (Executive Information Systems). In essence, these are sets of reports based on data from an enterprise information system. These systems in real time reflect the main aspects of production and financial activities, which helps to make operational decisions.

DSS of the second type suppose a sufficiently deep study of data, specially converted so that it is convenient to use them in the decision-making process. An integral component of such DSS are decision rules. Their technologies are based on the principles of multidimensional presentation and analysis of OLAP data (Online Analytical Processing) $[6,10,14]$.

In practice, the operational tasks of the project can be solved by one person or by a group of experts. The choice of one or another method depends on the specific features of 
the task, the number of project participants interested in the solution, as well as the ratio of costs and effect.

The strategic objectives of real estate development are solved exclusively collectively. And this is not by chance. The expenditure of resources and time to gather information, to fully prepare for making complex and expensive group decisions is always less than the averted loss from making the wrong, not the best or un worked decision [15, 16]. However, it is at this pre-investment stage that the negative effects of group decisions can have the most serious consequences. Therefore, it is during the working-out of a strategic plan for development that decision-making methods should be used, which allow minimizing the negative psychological effect of group work $[17,18]$.

Part of this problem already has its solution. In the early $80 \mathrm{~s}$, researchers at the University of Arizona developed the Group Decision Support System (GDSS).

The GDSS, also known as Electronic Meeting System, GroupWare, Computer Supported Co-operative Work, is a collection of software, hardware, and tools whose common goal is automated support for teamwork [19]. The first GDSS was based on a local area network that integrated personal computers located in the meeting room. Displaying the results of group work was carried out on a large screen, which focused the group's attention on the current issue.

The meeting included 3 phases:

1. Electronic brainstorming. Each of the participants (anonymously) collected and sent their ideas on how to solve the existing problem.

2. Analysis of ideas. Ideas were argued, criteria for evaluating alternatives were chosen.

3. Voting and data processing using various techniques.

To make a decision with the help of GDSS, a specialist is needed, leading the expert survey group. His role is key because he provides technical assistance, a moderator function and the role of a group decision-making session leader. These responsibilities at different stages of testing the system can be assigned to different people [20, 21].

The main advantage of using GDSS is the parallel, anonymous and structured communication between participants with error-free documentation. The fact that each participant writes and responds in parallel / asynchronously to the other members of the group can significantly save time and provide more information for analysis. A study by Ventana of the use of GDSS showed an increase in productivity of meetings at enterprises by $90 \%$ [22].

One of the major drawbacks of this system is the need for a qualified leader. His task is not only to be the moderator of the meeting, but also to choose the most appropriate methods, means and tools for solving the problem. From his work, in the end, depends on the result. His mistakes can completely distort the results [10].

The second drawback, which significantly complicates the use of this type of GDSS in the pre-investment phase of the real estate development project, is the need to gather all interested parties at the same time in the same room. Given the number of project participants and those interested in the project, it is not possible to fulfil this condition. Does this mean that the use of GDSS during the pre-investment preparation of the project of real estate development is impossible? We tried to answer this question during our research.

Research goal: to analyze the possibility of using existing group decision support systems in the early phases of a real estate development project and to assess the benefits of indirect computerized communication when making group decisions before direct communication during meetings. 


\section{Methods}

To achieve this goal, we used methods for analyzing literary sources, the functionality of existing decision support systems software presented on the Russian and world markets, and expert survey methods. Also, to identify the benefits of mediated parallel computerized communication in the process of making group decisions, the methods of observation and experiment were used.

The experiment (2017-2019) was attended by MBA students in the construction and real estate - the leaders of construction and development companies. The subjects were offered a problem situation: it is necessary to propose options for the redevelopment of an unfinished real estate and draw up a preliminary plan for the project.

The experimental group was divided into two subgroups. The first subgroup performed the task in the course of direct live communication in the classroom. The second subgroup interacted indirectly with the use of computer technology. The students of the second group were outside the classroom (distance learning format) and communicated with each other through chat. For the assignments, both groups were given 40 minutes.

\section{Results}

Analysis of the results of the experiment and observation in general confirmed our scientific hypothesis. Using written mediated communication reduces the time for discussion and decision making. Observing the decision-making process in the audience and analyzing the correspondence of participants communicating through group chat showed the following.

With direct communication:

- the initiative, as a rule, is in the hands of one person. He leads the discussion, makes the first proposals and criticizes the subsequent proposals of the discussion;

- If there are two or three active participants, the discussion turns into a confrontation between the parties, who do not always manage to agree among themselves;

- There is always at least one participant who does not participate in the discussion of the problem. His vote may not be taken into account when voting and choosing the best solution;

- During the discussion, participants are often distracted by extraneous topics, they recall stories from their own experience in the implementation of other development projects;

- At the end of the decision making time, only the participant who initiated the decision can justify the decision made;

- It is not always possible to reproduce the key points of decision making (especially if the protocol is not kept or is kept in a generalized form).

In indirect communication using computer technology:

- The work is more effectively structured if the moderator communicates with a voice, and the other participants express their opinions in writing. Less effective if everyone only communicates in writing;

- At the beginning of the discussion, participants seek to get as much information as possible - ask clarifying questions and answer them. The number of answers to a given question may be equal to or even exceed the number of participants. Some questions may remain unanswered. In parallel, several issues can be discussed. Sometimes answers are presented as links to external resources;

- Solutions are introduced and discussed in parallel, rather than sequentially;

- Individual participants may be distracted by extraneous topics, this does not prolong the discussion much, because processes run in parallel; 
- Some participants may not speak out on the questions asked and not take part in the voting;

- At the end of the discussion, participants can restore the decision-making logic (based on the available materials). The rationale for the decision, as a rule, is a short form without details.

Comparing the results obtained, we can conclude: the use of mediated communication using computer technologies effectively structures discussion of the problem and decision making. This reduces the time for discussion, because distractions to extraneous topics and ineffective debates are minimized, all the logic of the discussion is preserved. However, the positive effect of this type of work can be achieved only if there is a professional moderator who manages the discussion orally and not in writing.

As a result of an expert survey of MBA students, it was found that regardless of the size of a development company (from hundreds of employees to several thousand), the vast majority use a limited number of automated control programs. First of all, it is a postal service and corporate electronic document management systems: 1C, Tessa, PayDox, LanDocs. One of the functions of these programs is the meeting management function. It allows you to reduce the time for preparing the agenda and minutes, provide access to the current version of the agenda, speed up the search for documents related to meetings (presentations, minutes, etc.) and simplify the preparation of reports.

As we see, the maximum support that companies in the investment and construction sector can count on in making group decisions is a timely synchronous exchange of documents. Group decisions are made only as a result of live communication. The vast majority of our respondents did not even hear about the possibilities of using specialized computer systems to help make decisions based on the analysis of large volumes of information. At the same time, experts have noted the great potential for increasing the effectiveness of collective decisions with the help of DSS, especially when developing complex and large-scale development projects

An overview of the DSS software products on the market has shown that the most common are rapid response DSS (the first type of the products discussed above). Software systems TriniData, Simba DSS, SVIR-R and others provide operational decision support for the rational organization of production, load distribution.

The market leaders such as Oracle Collaboration Suite, Microsoft Exchange 2000, Sun Microsystems and Lotus Domino / Notes have developed Integrated Collaboration Environment. They remove one of the serious limitations of GDSS - the need for all participants in the discussion to be in one place. These systems allow the group to work with information in a mode distributed in time and space [6]. However, they do not include a base of group decision making methods. There are systems whose purpose is to support group decision making. But, the decision-making methods they use are limited to one method, for example, the rule of most - GroupSystems, MeetingWorks, or by hierarchy analysis method - ExpertChoice, or by pair comparison method - IGLA. Matlab math packages present a wide range of decision-making methods, but they do not support distributed group work.

The most interesting for use is the GDSS, described in the work of Dubrovinoy A.V. and Puginoy T.V. They describe the decision-making technology, the participants of which is a group of experts / specialists in the problem area. All the work of experts is carried out in anonymous mode. The authors believe that this ensures the honesty and unchainedness of the participants, the absence of a hierarchy of "boss - subordinate", reduces the likelihood of forming coalitions based on personal likes and dislikes. The process is led by a specially appointed moderator [10]. Its presence, as shown by our observation, increases the efficiency of the group decision making process. However, as mentioned above, it is the leader who can become the main cause of the erroneous decision. Performing the role of the 
Lead requires that a person has not only professional knowledge, but also strategic, moral, psychological and managerial abilities.

Thus, we were unable to find a single software package that would meet the features and requirements of the group decision-making process in the pre-investment phase of a real estate development project:

- a software package that allows participants with different expert characteristics to process large arrays of heterogeneous information;

- a software package that gives participants the opportunity to work distributed in time and space;

- software complex that overcomes the disadvantages associated with the subjectivity of human behaviour.

\section{Discussions}

In the field of real estate development, systematical decision-making support is required, since there cannot be without well-founded, logically demonstrable arguments and rationales for the decision made. To make decisions at the early stages of a development project, it is necessary to conduct detailed modeling of the consequences of the proposed decision, finding the optimal way to achieve a given result using simulation technologies. For this, various computer technologies are widely used [3].

However, no modern software complex is aimed at making decisions for a person. The task of artificial intelligence is to automatically process large volumes of information and present it in such a way as to make it easier for a person (project participants) to make an informed decision [2]. During the implementation of the development project, managers at various levels have to make many management decisions. Some decisions are made singlehandedly. Others - only in conjunction with other interested parties.

To avoid bias and advance the interests of one project participant, group decision making should be based on expert assessment of the problem. At the same time, the use of software built on mathematical methods of information processing can lead to the fact that, firstly, when processing and aggregating experts' preferences, their differences in views will be averaged, and as an effective solution, an option will be chosen, in the assessments of which the opinions of experts turned out to be more or less agree with each other. Secondly, the individual opinion, which may constitute the "exceptional" knowledge of an expert, may be smoothed out, lost. At the same time, experts who disagree with the majority are simply ignored [6]. However, it is often impossible to assess the validity of different, and sometimes diametrically opposed points of view of experts on one problem. The opinion of one expert will one way or another reflect the interests of one of the stakeholders. Hence the inevitability of the use of computer decision support systems. Currently there is no product on the market that would allow to overcome all the negative aspects of a group decision. Therefore, development companies are forced to use different Decision Support System at different stages of work, and the final decision is still made in an authoritarian way or through open discussion.

\section{Conclusions}

Thus, the group decision support system (GDSS) system is a promising and relevant direction in the evolution of decision support system (DSS). Despite the fact that evolution may require significant investments, the organizational benefits of implementing it in a development company can be significant and substantial. 
This work was financially supported by Ministry of Science and Higher Education of the Russian Federation (\#NSh-3492.2018.8).

\section{References}

1. E.A. Gusakova Organizational and technological genesis as a tool for strategic planning of large-scale real estate development projects, Business Technologies 2018"MATEC Web of Conferences, 6 (2018)

2. A.V. Ginzburg Information model of the life cycle of a construction object, Industrial and Civil Construction, 9, 61-65 (2016)

3. E.A.Gusakova, E.V. Romanova Real estate project management: models of interaction of participants, / International scientific and technical magazine "Real Estate: Economics, Management". - Moscow, MGSU, 4, (2018)

4. T. Kuzmina, S.A. Sinenko, A. Slavin A 2016 Combining the functions of the main participants of investment and construction activities at the present stage, Industrial and civil construction, 6, 71-75 (2016)

5. A.I. Pavlov, A.Yu. Yurin, G.S. Maltugueva Decision Support System for Group Selection Problems, Software Products and Systems, 2, 54-57 (2011)

6. T. K. Kravchenko Making Group Decisions Using Expert Decision Support System, Information Technologies in Design and Production, 2, 68-75 (2015)

7. A.A. Zakharova, O.A. Kolegova Development of the structure and functions of the standard module "work with experts" for the decision support system of the strategic management of the organization, New research in the development of technology and technology, 2, 18-2 (2017)

8. A .V. Ginzburg, O. Baranova, N. Blokhina, A.A.Volkov, N. Garyaev, V. Ignatov, E. Ignatova, B. Istomin, P. Kagan, E. Kitintseva, V. Kulikov, S. Sinenko Systems of design automation in construction (Moscow: Moscow State University of Civil Engineering, 2014)

9. A.V. Ginzburg A. Skiba Creating an urban area planning design based on the theory of fuzzy logic, Applied Mechanics and Materials Vols 584-586, 507-511 (2014)

10. A.V. Dubrovina., T.V. Pugina Group decision support system, Radio electronics, informatics, management 1(11), 145-149 (2004)

11. Yu.V. Bugaev, M.S. Mironova, B.E. Nikitin, A.S. Tchaikovsky Decision Support System based on extrapolation of expert estimates using the maximum likelihood method, Bulletin of Bryansk State Technical University 1 (25), 84-90 (2010)

12. A.V. Ginzburg and E. Nesterova The technology of continuous information support for the life cycle of a construction site, Scientific and Technical Journal Vestnik MGSU. 5, 317-320 (2011)

13. I. Segaev, A. Porshakova, A.Vakina, O. Petrosova Modern problems of development and realization of complex residential development of territories, Economics and entrepreneurship 10 (51), 305-310 (2014)

14. V.I. Dubrovin, N.A. Mironova Intellectual information technology of a choice of a method of acceptance of group decisions, Control systems and information technologies T. 53. 3-1, 133-136 (2013)

15. E.A. Trakhtengerts Computer management decision support systems, Management problems 1, 13-25 (2003)

16. I.A. Kirikov, A.V. Kolesnikov, S.V. Leaf Fall, S.B. Rumovskaya Virtual heterogeneous teams supporting decision making, Systems and IT tools T. 25. 3, 126149 (2015)

17. A.N. Raikov, A.V. Ustinov, A.G. Chikina Acceleration of reaching agreement in team decision-making in a network environment, Defense Complex - to the scientific and 
technical progress of Russia 3(127), 3-9 (2015)

18. O.A. Zhirkov, A.V. Ivaschenko, A.Yu. Smirnov, V.A. Shcherbakova, A.S. Shtul Solving semistructured problem situations using intellectual systems, In a collection of reports and scientific papers of the II International Forum (conference): Corporationsparadigm of national economy formation 329-338 (2014)

19. E. Romanova, K. Mochkin and E. Rakhmankulova Application of computer decision support systems in realization of development projects at pre-investment phase, Economics and entrepreneurship 11(100) 847-850 (2018)

20. A.V. Andreichikov, D.S. Markov, L.A. Astashkina Intellectual system for strategic decisions, World of Transport T. 12, 5 (54), 130-14 (2014)

21. J. Wall, M. Bertoni, T. C. Larsson ICED, 4, 513-522 (2017)

22. J. N. D. Gupta, G. A. Forgionne, M. T. Mora, Intelligent decision-making support systems : foundations, applications and challenges (London . Springer, (2006) 